\title{
Dai liquori casalinghi ai liquori della tradizione: il caso delle Valli di Lanzo
}

From homemade liqueurs to traditional liqueurs: the case of the Lanzo valleys

\section{Gianpaolo Fassino}

\section{OpenEdition}

Journals

Edizione digitale

URL: https://journals.openedition.org/aam/4705

DOI: $10.4000 /$ aam.4705

ISSN: 2038-3215

Editore

Dipartimento Culture e Società - Università di Palermo

Notizia bibliografica digitale

Gianpaolo Fassino, «Dai liquori casalinghi ai liquori della tradizione: il caso delle Valli di Lanzo», Archivio antropologico mediterraneo [Online], Anno XXIV, n. 23 (2) | 2021, online dal 31 décembre 2021,

consultato il 09 janvier 2022. URL: http://journals.openedition.org/aam/4705 ; DOI: https://doi.org/

$10.4000 /$ aam. 4705

Questo documento è stato generato automaticamente il 9 janvier 2022.

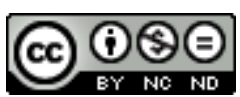

Archivio antropologico mediterraneo è distribuita con Licenza Creative Commons Attribuzione - Non commerciale - Non opere derivate 4.0 Internazionale. 


\title{
Dai liquori casalinghi ai liquori della tradizione: il caso delle Valli di Lanzo
}

From homemade liqueurs to traditional liqueurs: the case of the Lanzo valleys

\author{
Gianpaolo Fassino
}

1 1. I liquori d'erbe, i rosoli, il ratafià, la grappa, per non dire del fernet e del vermouth, sono elementi che concorrono in maniera importante a determinare il patrimonio gastronomico piemontese. Mentre per il vino (Berta, Mainardi 1997), la grappa (Cipolla, Damonti 2019) e il vermouth (Mainardi, Berta 2018) si è ormai consolidata una ricca tradizione di studi, e relativa bibliografia, la produzione liquoristica è rimasta sinora un po' ai margini rispetto al più generale interesse del pubblico e degli studi verso il patrimonio gastronomico regionale.

2 Partendo da questa consapevolezza si è avviata la ricerca di cui diamo conto, un'indagine che porto avanti dal 2016 nel quadro di un mio più vasto interesse sulle forme e le pratiche di consumo dei generi voluttuari (caffè, grappe, liquori, cioccolato, ecc.) all'interno della società italiana (Fassino 2020). Un'indagine che ha mosso i suoi primi passi all'interno di un seminario metodologico organizzato presso l'Università degli Studi di Scienze Gastronomiche ${ }^{1}$ per poi proseguire successivamente presso l'Università del Piemonte Orientale, nell'ambito della ricerca L'integrazione del patrimonio e delle risorse locali nelle filiere turistiche della montagna debole italiana condotta nel 2020-21 (progetto Italian Mountain Lab - Ricerca e Innovazione per l'ambiente). Dopo una prima fase volta a indagare la produzione del vermouth - la cui ricetta ha come componente essenziale le erbe officinali - l'indagine è proseguita e si è ampliata con lo studio dei liquori della tradizione piemontese. Il quadro metodologico di riferimento si è altresì avvalso delle esperienze maturate nel corso del progetto Interreg Central Europe SlowFood CE - Culture, Heritage, Identity and Food (Grimaldi, Fassino, Porporato 2019). La ricerca, parte di un più complessivo piano di lavoro e di cui si vuole offrire in questa sede un primo resoconto, è stata condotta attraverso la raccolta di una serie di interviste qualitative a operatori della filiera liquoristica (coltivatori di erbe, maestri 
liquoristi-distillatori, consumatori, ecc.) nonché attraverso la raccolta e analisi di dati storici e quantitativi. Le testimonianze raccolte sono depositate presso il Laboratorio di antropologia visiva dell'Università del Piemonte Orientale e sono in parte rese disponibili attraverso l'archivio multimediale dei "Granai della memoria"2.

3 2. La preparazione di liquori e distillati è storicamente attestata un po' in tutto il territorio regionale, sia a livello domestico che artigianale. Nelle case delle famiglie del «vecchio Piemonte» - ma siamo di fronte a un tratto comune dell'intera società italiana - era sempre presente una bottiglia di grappa o di liquore da offrire agli ospiti o da consumare, nei giorni di festa, a fine pasto, come suggerisce il poeta Ergasto Acrivio: «dopo tavola il caffè / e rosoli bianchi e neri» (Le villeggiature 1794, cit. in Camporesi 1998: 29), consumati sotto l'incombere di un "gran lampadario vetusto che pende a mezzo il salone / e immilla nel quarzo le buone cose di pessimo gusto» (Gozzano 1911: 88).

4 La letteratura sul gentiluomo di campagna e i ricettari a stampa contemplano sovente, al proprio interno, una sezione dedicata alla produzione di liquori e distillati, segno e traccia di un interesse che la borghesia subalpina - cui erano indirizzate in primis queste pubblicazioni - riservava a questi prodotti. L'avvocato ossolano Jacopo Albertazzi nel dare alle stampe, sul finire del Settecento, la fortunata opera Il padre di famiglia in casa ed in campagna (Vercelli 1790-91) si soffermava ad esempio sulla produzione di acquavite e rosolio. Proprio a proposito di quest'ultimo ne sottolineava l'eccellenza: «Il rosolio però della nostra dominante [Torino] è quello che porta il vanto ne' nostri contorni, tanto perché procedente da vini di ottima pianta, quanto perché si sa in essa ottimamente l'arte di distillare, quantunque io creda che molto v'influisca l'aria» (libro VI, cap. II, par. 63). L'alta nomea del rosolio torinese e degli altri liquori subalpini trova conferma nei resoconti di viaggio del Grand Tour. Il geografo britannico Thomas Salmon nell'opera Lo stato presente di tutti i paesi, e popoli del mondo, pubblicata nel 1740 , confermò «l'eccellenza dei rosolii e dei liquori torinesi e la stima di cui venivano meritatamente circondati» mentre le Nouvelle instruction pour les confitures, les liqueurs, les fruits pubblicate a Parigi a inizio Settecento registravano la ricetta di un «Rosolio di Torino», segno di una circolazione reciproca di ricette fra Piemonte e Francia (Viriglio 1898: 176; Arpino, Antonetto 1990: 71; Doglio 1995: 266).

5 I numerosi ricettari regionali, molti dei quali ancora di derivazione francese (Rosso 2009), editi a Torino fra la seconda metà del Settecento e la metà dell'Ottocento confermano e codificano ulteriormente questa tradizione liquoristica regionale. Si tratta di testi - è bene rimarcarlo - in cui venne definito e consolidato un modello di gastronomia regionale, un vero e proprio canone del mangiar bene piemontese in cui i liquori completano e sigillano un pasto ideale, un «disné da spos» («un pranzo di nozze») fatto di numerosi antipasti, agnolotti, fritto misto, bollito, brasato, finanziera, carpione, bagna caoda, ... (Ruggero 2007: 116).

6 Ricco di indicazioni sulla preparazione dei liquori è Il cuoco piemontese perfezionato a Parigi, stampato a Torino nel 1766 (ma di derivazione parigina, calcando pesantemente La cuisiniere bourgeoise di Menon; Dickie 2007: 218), il quale dedica un intero capitolo ai «siroppi» e un altro ai «frutti all'acquavita e rattafia» (Serventi 1995: 282-286, 291-296). Una scelta ancor più ampia è contenuta nei Raccoglimenti di varie sorti di confiture praticate dal confitturiere di Sua Maestà Sarda, un manoscritto di fine Settecento (Gattullo 2002) ricco di sciroppi di frutta e di un paio di ricette di vin brulé, così come una buona scelta di distillati, elisir, liquori è presente nella raccolta intitolata Il confetturiere 
piemontese (1790). Una scelta che ritroviamo, ulteriormente arricchita, nella summa della ricettistica subalpina, il monumentale Trattato di cucina, pasticceria moderna, credenza e relativa confettureria di Giovanni Vialardi (1854) in cui un capitolo è riservato a «liquori e ratafià per filtrazione ad uso di famiglia» e un altro a «Dei siroppi», anch'essi "ad uso di famiglia», dicitura che sottolinea e conferma il carattere domestico e casalingo di queste preparazioni (Vialardi 1854: 570-578, 588-592). Un coevo ricettario francese, di cui comparve nel 1843 un'edizione torinese - intitolato La cuciniera di città e di campagna o nuova cucina economica - proponeva ai lettori piemontesi svariate ricette di sciroppi e ratafià, ma anche di anisette, maraschino, punch. Una varietà di preparazioni che si sarebbe ulteriormente arricchita due anni dopo con la pubblicazione di uno specifico ricettario: L'arte di conservare e di adoperare le frutta che contiene tutti i metodi più economici per confezionare e comporne liquori, sciroppi, gelati, bevande domestiche e simili (1845).

La pratica di preparare in casa i liquori - sfruttando ad esempio le piante da frutta della cascina, ma anche «le erbe officinali, radici o semi» (Cavallero 2005: 137) - trova conferma nella ricorrente presenza di queste ricette nei quaderni manoscritti: «i cari vecchi "quadernetti" neri delle massaie e delle padrone di casa: mogli meno frettolose delle nostre - chiosava il gastronomo Giovanni Goria - si annotavano nelle ingiallite pagine a quadretti piatti di cucina, conserve e marmellate, rosolii e liquori tipici, quasi esclusivi della loro specifica casata» (Goria 1978: 6). Quaderni in cui - ha evidenziato Piercarlo Grimaldi - le donne di campagna «compitavano e tracopiavano le singole ricette», fragili e sporadiche tracce di una «non ancora scritta storia gastronomica» (Grimaldi, Fassino 2017: 56). Arquebuse, ratafià, liquore di violette, nocino, cento erbe sono alcuni dei nomi ricorrenti che è possibile ritrovare sfogliando i ricettari domestici del Piemonte rurale (Caire 1978: 153-157, 160-161). Attingendo al mio archivio di famiglia, con la grafia della mia nonna paterna, ritrovo ad esempio la ricetta di un liquore al caffè:

Caffè Moka 2 etti

Caffè Portorico 1 etto

$1 \mathrm{Kg}$. di zucchero

Mezzo Kg. di spirito finissimo

3 bastoni di vaniglia

Si fa il caffè con due litri di acqua quindi si mette lo zucchero e si fa bollire molto adagio quando ha finito di schiumare si toglie dal fuoco e si lascia raffreddare. Si aggiunge lo spirito e si imbottiglia. Si taglia la vaniglia in pezzetti che si mettono un po' per bottiglia quando queste sono piene. Poi si tura.

Scartabellando un quadernetto di ricette di Enrica Costa, una donna della piccola borghesia torinese, ecco invece comparire il «rosolio d'arancio», lo «sciroppo d'amarene» e l'esotico «sciroppo tamarindo», quest'ultimo lascito dei rapporti coloniali dell'Italia con l'Etiopia e la Somalia. Attraverso la circolazione dei ricettari manoscritti e a stampa - e la reciproca frequentazione fra classi popolari e borghesi - si pensi al ruolo delle domestiche (archetipo torinese ne è la Natalina di Lessico famigliare), donne contadine inurbate a Torino per servire la borghesia cittadina - si crea una feconda contaminazione e reciproco scambio di pratiche culinarie, di usi e abitudini gastronomiche fra le «culture egemoniche» e le «culture subalterne» (Cirese 1973). Il confronto fra il quaderno manoscritto di Enrica Costa e quello di mia nonna - che ne fu per due decenni la sua donna di servizio a Cit Turin, nel cuore di Torino - evidenziano questa reciproca dipendenza. Mia nonna, figlia di una modestissima famiglia mezzadrile, nei suoi quasi vent'anni di vita torinese (1928-1946) aveva acquisito, sotto 
la guida materna e sapiente di Enrica Costa, straordinarie competenze gastronomiche che riportò, una volta rientrata nelle natie colline astigiane, nella quotidianità della sua pratica culinaria: un tratto distintivo rispetto alle sue sorelle che erano rimaste nella cascina, senza mai allontanarsi troppo dal paese natio. Un modello di circolazione delle competenze già descritto e confermato dagli storici dell'alimentazione (Capatti, Montanari 1999: 273-284; Montanari 2001) e che ben si adatta - per il caso piemontese alle tecniche di preparazione di sciroppi e liquori. La diffusa presenza in passato, anche nelle case contadine, di rosoli, nocini e liquori d'erbe derivava in misura significativa da questa vasta circolazione di saperi empirici fra classi sociali diverse. Erano liquori che all'elemento edonistico sovente abbinavano, nella farmacopea popolare, un valore curativo come nel caso del fernet (usato addirittura in occasione dell'epidemia di Colera del 1867), del Toccasana Negro (nomen omen) o di molti sciroppi ed elisir (Doglio 1995: 115, 128, 149; Ruggiero 2007: 105, 158; Piccinino 2016; Carpignano 2017: 144, 178-179).

9 Attraverso l'autoproduzione domestica si poteva così avere, anche nelle case e famiglie più modeste, una piccola scelta di buoni liquori «con costi minimi» (Cavallero 2005: 137; Vaudagnotto Bianco 2010: 159). Si producevano «liquorini squisiti» a partire da frutti e bacche, come nel caso del sambuco o del prugnolo selvatico, piante spontanee poi dimenticate (Bocchino 1988: 48-49). Questa pratica dell'autoproduzione dei liquori che era stata autorevolmente sancita anche da La scienza in cucina di Pellegrino Artusi e poi ulteriormente confermata dal Talismano della felicità di Ada Boni - è andata parzialmente scemando nel corso del Secondo dopoguerra, parallelamente al diffondersi di nuovi stili di consumo all'interno della società italiana (Scarpellini 2012: 175-185; Cavazza, Scarpellini 2018) e allo svilupparsi di un'industria liquoristica nazionale che riusciva a distribuire capillarmente i propri prodotti, sostenendoli anche con fortunate e pervasive campagne pubblicitarie. L'etnografo abruzzese Francesco Galiffa, studiando il vin cotto teramano, ha evidenziato come proprio nel contesto del boom industriale si sia registrata una cesura storica, con l'interruzione dell'autoproduzione domestica dei liquori a partire proprio dagli anni Sessanta, quando «la bottiglia di vino cotto e pure quelle degli altri liquori fatti in casa scomparvero dalle [...] credenze delle cucine di campagna per essere sostituite, nelle vetrine delle sale, dai liquori simbolo della civiltà industriale, veicolati nelle case attraverso Carosello: la sambuca Molinari, l'amaro Cynar, lo Stock 84 ed altri ancora» (Galiffa 2013: 103). Una parabola avviatasi negli anni Cinquanta, consolidatasi nel corso del decennio successivo e che ha conosciuto poi il suo apice negli anni Ottanta con la «Milano da bere», icastica pubblicità dell'amaro Ramazzotti entrata nell'immaginario nazional-popolare: uno stile di consumo in cui «non compriamo liquori, ma status sociale» (Scarpellini 2006: 22; Venè 1990: 233). Massimo Alberini, nel suo Piemontesi a tavola segnalava come sul finire degli anni Sessanta del Novecento fosse ormai tramontato l'uso di produrre in casa «certi liquori piemontesi oggi caduti in disuso, come i rosoli, gli alchermes e i noti tipi di ratafià al ginepro, di noci, di lamponi» (Alberini 1967: 235). Un fenomeno che parallelamente colpì, in quello stesso torno di anni, le piccole realtà produttive, come testimonia Carlo Quaglia, classe 1971, alla quarta generazione di una famiglia di liquoristi-distillatori, che così narra la storia della distilleria di Castelnuovo don Bosco, sulle colline astigiane:

l'azienda è nata nel 1890, diventata di proprietà della famiglia nel 1902, comprata da mio bisnonno, un personaggio eclettico, investiva in tanti settori, aveva deciso anche di acquistare questo tipo di attività [...]. Ai tempi l'azienda produceva veramente grosse quantità di grappa che venivano, oltre che imbottigliate 
internamente all'azienda, vendute anche sfuse ad altre realtà produttive. Tra le due guerre, oltre alla produzione della grappa, si è iniziato a produrre anche tutta una gamma di liquori, amari, elisir e i classici cordiali che si consumavano in quegli anni, si è poi passati anche alla produzione di vini aromatizzati: il classico barolo chinato e i vermouth, che però poi sono decaduti negli anni Settanta, vuoi perché l'attenzione del pubblico si è spostata su prodotti d'importazione come whisky, cognac, armagnac, liquori dolci esteri e la parte di aperitivo nazionale ha subito una lieve flessione e soprattutto le produzioni si sono spostate su prodotti a basso costo, proprio perché la concorrenza iniziava ad esserci, bisognava essere competitivi, a discapito però delle rese qualitative del prodotto finito, quindi con un decadimento totale della fascia di consumo, dove alcune multinazionali, grosse aziende si sono affermate come brand principale e le piccole aziende produttrici hanno cessato la produzione ${ }^{3}$.

10 Fra i pochi liquori a godere in Piemonte di una continuità di produzione anche nel corso degli anni Sessanta e Settanta va annoverato il ratafià di Andorno, ricavato dalle ciliegie selvatiche grazie all'impegno della famiglia Rapa, titolare dell'omonimo liquorificio, e alcune produzioni di genepy (Alberini 1967: 235; Veronelli 1968: 20, 176; Perera 2001: 100-110).

11 3. In questo quadro assumono una nuova rilevanza anche i liquori alle erbe alpine, gli estratti di artemisia, di ginepro e genziana (Biasiol 1974: 120-122; Marchese 1998: 200-202; Ruggiero 2007: 157-159) il cui consumo nel corso degli ultimi è tornato, in maniera vieppiù significativa, ad essere presente nell'orizzonte gastronomico piemontese. Se ne trova traccia significativa negli elenchi delle bevande via via inserite, a partire dal 1999, negli elenchi dei prodotti agroalimentari tradizionali del Piemonte, nella categoria delle «bevande analcoliche, distillati e liquori», in cui attualmente compaiono il bicerin, il Garus susino, i liquori di erbe alpine, il Nocciolino di Chivasso, l'olio essenziale di menta piperita, il ratafià, il rosolio e il vermouth. A questi, nel corso degli anni si sono affiancati negli elenchi dei PAT regionali altri prodotti, quali l'arquebuse (o Alpestre), il genepy e alcuni elisir, che poi, per svariate ragioni, sono usciti dal catalogo ufficiale (ad es. il genepy in quanto nel frattempo ammesso a fregiarsi dell'Indicazione Geografica, menzione tutelata dalla normativa europea).

Appare utile dunque approfondire il fenomeno della riscoperta dei liquori tradizionali piemontesi da parte dei consumatori. È in questo utile ricorrere ancora alle parole di Carlo Quaglia, informatore-chiave, grazie al suo osservatorio privilegiato di liquoristadistillatore, ricco anche dell'esperienza della propria famiglia, impegnata in questo settore da oltre un secolo:

La riscoperta è generazionale: i nostri nonni conoscevano benissimo il kummel, il liquore fatto con i semi del cumino dei prati, conoscevano benissimo l'elisir di camomilla, i cordiali, i rosoli, erano tutti liquori consumati abitualmente in casa: veniva l'ospite e c'era sempre il bicchierino di liquore che accompagna la pasta secca o dopo il caffè [...], dimenticati dopo i nostri nonni, sono mancate due generazioni fondamentalmente a consumare questo tipo di prodotto a scapito di altri superalcolici d'importazione. Il riproporli è stato anche facile: ti poni di fronte a generazioni che si stupiscono, non conoscono e non hanno mai assaggiato un liquore di cumino, un liquore di camomilla, un liquore di agrumi freschi, e questo stupisce, stupisce sempre... siamo stati abituati negli anni Ottanta e Novanta al prodotto industriale, grandi spot, consumo indotto, ... l'avere il liquorino della nonna finita la nonna era impossibile, l'abbiamo reso fruibile nuovamente [...]. Abbiamo rispolverato le tradizioni italiane, quello che era un prodotto storico. partire dalle basi vegetali raccolte nelle Valli di Lanzo, nelle Alpi Piemontesi. Si tratta di 
una serie di liquori realizzati dalla Distilleria Quaglia: un'azienda che, seppur non situata in territorio montano, ha trovato nelle Alpi Graie il proprio territorio di riferimento non solamente per quanto concerne l'approvvigionamento delle materie prime, ma in quanto parte di un più complessivo progetto di sviluppo territoriale. Una sinergia che si inserisce a pieno titolo nelle pratiche virtuose che possono riorientare dopo decenni di abbandono delle terre alte italiane - ad un «ritorno alla produzione nelle "montagne di mezzo"», produzione che non può essere chiusa all'autoconsumo ma aperta, embricata «con relazioni orizzontali legate a commerci e scambi» (Varotto 2020: 113), così come peraltro era storicamente, in cui le produzioni della montagna erano parte di un vasto sistema di scambio e mercato con la città, la pianura e la collina. Proprio con le comunità delle Valli di Lanzo è ad esempio storicamente documentato un intenso scambio commerciale volto all'acquisizione dei vini prodotti nelle colline dell'Astigiano e del Monferrato (Clavarino 1867: 277).

Il caso che stiamo analizzando rientra nel quadro di quella che Mauro Varotto ha definito «terza montagna», un nuovo spazio alpino che «si sta facendo spazio, dopo la "prima" montagna estremamente povera e ancorata a sistemi di magra autosussistenza nel periodo di massima pressione demografica, e una "seconda" montagna appendice della pianura e asservita a modelli produttivi industriali», una montagna in cui la sfida è quella «di un nuovo modello di pensiero che sta lentamente emergendo: quello della polifunzionalità degli interventi, del rispetto del contesto che si trasforma in specificità colturale e protezione ambientale» (Varotto 2020: 115).

L'avvio di una specifica produzione e valorizzazione di liquori prodotti a partire dalle erbe coltivate e raccolte nelle Valli di Lanzo da parte della Distilleria Quaglia è peraltro parte di un più vasto progetto aziendale di riscoperta dei liquori tradizionali che ha il proprio cuore nella linea 'Vintage' il cui claim annuncia «liquori moderni nati da una tradizione antica». Si tratta di una serie di liquori che ben avrebbero potuto figurare nel gozzaniano salotto di nonna Speranza: l'Assenzio, il Bergamotto, la Camomilla, il Chinotto, il Doppio Carvi (a base di semi di cumino), il Fernet, il Pino Mugo, la Liquirizia, il Rabarbaro, il Sambuco, gli estratti di rose e viole, prodotti confezionati fra l'altro in una bottiglia dal design liberty che, anche nel packaging, richiama alla memoria un salotto protonovecentesco. Si tratta di liquori che fanno parte a pieno titolo della tradizione liquoristica italiana (Odello et al. 2013) e che incorporano la storia della famiglia Quaglia. La riscoperta e rimessa in produzione dei liquori tradizionali da parte della distilleria di Castelnuovo don Bosco è infatti avvenuta a partire dalla attenta rilettura dei «quadernetti neri» (Goria 1978: 6), «scritture non comuni» (Caffarena 2016) in cui le prime generazioni di distillatori della famiglia avevano annotato, nel corso della prima metà del Novecento, le ricette dei propri prodotti:

ogni distilleria storica ha i suoi quaderni segreti, generazionali; io oggi sto scrivendo quaderni che magari guarderanno i miei nipoti tra trenta-quarant'anni; io sto guardando quelli di mio bisnonno e di mio nonno. L'evoluzione è costante per ragioni di mercato, il quaderno da una descrizione quantitativa del prodotto e di metodologia, la metodologia logicamente cambia, cambiano le attrezzature: se prima avevi una pressa manuale, il torchio in legno, [...] oggi hai delle presse più dinamiche, più veloci, o più soffici, che ottimizzano, si può torchiare ad acqua, a pneumatico o meccanico, con delle pressioni molto alte. A volte si guardano questi quaderni, si guarda anche solo la lista quantitativa di ingredienti e si capisce già che quel prodotto non potrà mai funzionare, vedi delle quantità di genziana, o di china, o di calamo aromatico, o di assenzio che sono impensabili da usarsi ai giorni nostri... no, no..., degli amaricanti pesantissimi, molto molto intensi e da subito si 
parte con un po' di ricerca e sviluppo e si fa una prova, già abbassando questi aromatizzanti, dimezzandoli, è quindi sostanzialmente tutta sperimentazione: si guarda, si decifra, si fa una prova dal vivo, materiale, si degusta e poi si riprova e si corregge e si va avanti così, a tentoni, fin quando non si raggiunge un equilibrio e una compostezza del prodotto. È l'unico modo che abbiamo. Non abbiamo altro che l'esperienza e le prove. A nostra volta stiamo arricchendo il patrimonio aziendale di scritture, distinte base, quaderni, metodologie di produzione, siamo alla quarta generazione... fa piacere vedere che il bisnonno aveva preso da un francese alcune ricette, le aveva modificate, scriveva "ricetta modificata", quindi diventava la propria. Non c'era mai la regola, non c'è uno standard da seguire, è il palato del liquorista che può fare veramente la differenza in questi casi, è l'olfatto, la vista, ognuno lo fa su misura: è come essere dei sarti, costruire qualcosa che piace a te, poi se piace anche agli altri ben venga, il prodotto è identitario, anche un liquore può essere identitario, può esprimere qualcosa di chi l'ha fatto, un suo gusto: quando riesci sono sempre soddisfazioni.

La disponibilità di un patrimonio di ricette attinte o comunque ispirate dai quaderni manoscritti del nonno e del bisnonno e la lunga continuità aziendale, con oltre un secolo di storia alle spalle - «Liquori artigianali piemontesi dal 1890» recita un claim sul sito web dell'azienda - sono elementi preziosi che contribuiscono a costituire e rafforzare il capitale simbolico della «Antica Distilleria Quaglia». L'impiego poi di moderne metodologie di produzione che si innestano su consolidate conoscenze e competenze tradizionali fanno sì che la qualità del prodotto raggiunga oggigiorno standard molto elevati, rispondendo pienamente alle esigenze contemporanee. Carlo Quaglia così racconta il percorso che lo ha portato a riscoprire e reinterpretare, all'inizio del nuovo Millennio, i liquori che la distilleria già produceva nei primi decenni del Novecento e che poi erano usciti dalla produzione, surclassati da nuovi stili di consumo e dal successo di bevande dozzinali proposte dalle grandi imprese nazionali e internazionali:

Qualche anno fa ci siamo posti il problema di fare qualcosa di innovativo che non ci fosse ancora sul mercato. L'idea spontanea che è nata è stata quella di rispolverare vecchie ricette di famiglia, di un secolo addietro, rivisitandole in chiave moderna, riproponendole con formule adeguate ai consumatori attuali. Il lavoro è stato lento e lungo, è stato comunque molto efficace: c'è stato un restyling delle ricette e anche di tutto il packaging e di tutto il confezionamento, cosa molto importante nella vendita oggi. L'immagine nel settore degli spirits e dei superalcolici è determinante per la riuscita e il successo di un prodotto. Le ricette sono state alleggerite sia dal punto di vista del grado alcolico sia dal punto di vista degli aromatizzanti presenti nel prodotto finito, proprio perché cinquant'anni fa, settant'anni fa, ottant'anni fa si cercava un grado alcolico molto alto, il prodotto doveva essere bruciante, oggi si ha una tendenza a consumare prodotti a minor tenore alcolico, gli zuccheri sono stati ove necessario ridotti e le parti aromatiche, in controtendenza a quello che è l'attuale mercato, sono state portate da natural-identiche o sintetiche che dir si voglia a naturali.

Le occasioni di consumo di questi prodotti della tradizione oggigiorno non sono più solamente quelle di un tempo: dal salotto di nonna Speranza si è ora passati alla mixology e al bartending, utilizzando i liquori vintage quali ingredienti di originali aperitivi. I liquoristi e i distillatori devono tener conto, nella rivisitazione e preparazione dei prodotti tradizionali, anche delle nuove modalità con cui - almeno in parte - i liquori sono oggi consumati. La miscelazione necessita infatti di accorgimenti particolari che il consumo in purezza non richiedeva. Come spiega Federico Ricatto, maestro liquorista: 
c'è in questo momento un ritorno ai gusti più amari, come si usava circa settanta, ottanta anni fa, anche se amari non esageratamente come allora, si è lasciata indietro tutta la parte di miscelazione molto dolce, adesso mentre si lavorano molto meno i prodotti dolci, adesso per un po' di anni ci sarà un po' di ritorno all'amaro, e poi quasi sicuramente si tornerà di nuovo un po' verso il dolce: è la ciclicità dei prodotti. [...] abbiamo ad esempio riproposto il fernet: la parte amaricante nei cocktail oggi piace ${ }^{4}$.

18 Anche in questo fenomeno trova conferma il noto assunto che vuole la tradizione non già come un elemento statico, un culto delle ceneri, bensì come una «innovazione ben riuscita» (Montanari 2004: 10).

Siamo quindi andati a cercarci delle eccellenze territoriali italiane - prosegue Carlo Quaglia nel suo racconto - ed abbiamo lavorato materie prime di origine vegetale, possibilmente raccolte in zone vocate, direttamente dalle aziende agricole, quindi saltando la filiera distributiva e le possibili manipolazioni e variazioni qualitative che una filiera tradizionalmente può dare, cercando di usare veramente il fresco appena colto. L'estremo è stato l'autoprodursi alcune parte aromatiche coltivate in proprio, da me, raccolte ed essiccate o usate fresche presso la nostra azienda. I risultati sono stati ottimi, il riscontro è stato immediato, la visibilità grandissima, questo ci ha portato [...] ad aprire mercati esteri importanti, con grande soddisfazione personale.

19 È in questo contesto che si inserisce l'avvio di una significativa filiera produttiva di parti aromatiche nelle Valli di Lanzo, in particolare a Balme (Val d'Ala), come spiega ancora Quaglia nella sua testimonianza:

Muovendo direttamente un rapporto con il produttore agricolo riusciamo a sollecitare anche le persone che abitano in quei posti - vedi il caso del genepy, o di tutte le parti aromatiche che derivano dalle conifere che raccogliamo in montagna, le artemisie, [...] - dove prima c'erano i classici impieghi tradizionali: pastorizia, un po' di turismo [...]. Hanno capito che dedicare del tempo alla loro terra è anche redditizio, non è sempre e solo pascolo quello che vedi, può diventare qualcosa di più produttivo, di più redditizio. Abbiamo creato dei microredditi, abbiamo rilanciato delle piccole aziende agricole che all'inizio erano magari titubanti, in realtà quando hanno visto il primo raccolto-emissione della prima fatturapagamento sono stati molto soddisfatti. Magari prima per fare lo stesso utile in latte vaccino, piuttosto che patate, facevano una fatica incredibile, invece ora con una piantagione di genepy - meno fatica, tempi ristretti - si hanno dei risultati decisamente più importanti, e quindi c'è stato tutto un rilanciare il territorio con intervento di abitanti indigeni e con intervento di gente che dalla bassa valle è risalita e ha riconvertito terreni abbandonati a colture redditizie. Stiamo creando una piccola comunità di agricoltori che ci seguono e ci aiutano moltissimo. Quando hai bisogno dei fiori di sambuco, delle ciliegie selvatiche, dell'assenzio spontaneo, della genziana fresca sai a chi rivolgerti. Il valore aggiunto è altissimo a livello umano e anche la qualità è altissima: una genziana raccolta fresca e infusa entro le 24 ore, non essiccata, non manipolata, ha dei sentori decisamente più delicati rispetto alla genziana secca, una resa sul liquore finito diversa, una compostezza aromatica molto più intensa e molto più gradevole, questo ci rende abbastanza unici, la grande industria tendenzialmente ottimizza i costi, ottimizza perlomeno la produzione, ma la materia prima è sempre di origine sconosciuta e si va a comprare al ribasso. Noi cerchiamo la primizia, l'eccellenza del vegetale, quindi l'epoca di raccolta della ciliegia in piena maturazione, il lampone in piena maturazione.

Balme, posto al termine della Valle di Ala, al confine con la Francia, a oltre sessanta chilometri dal capoluogo torinese è un piccolo comune montano che ha conosciuto nel corso del Novecento, come gran parte della montagna italiana, un fortissimo spopolamento, passando dai 431 abitanti del 1861 ai circa 100 attuali (Castagneri, 
Sguayzer 2010). Balme, per questioni non solamente altimetriche e orografiche, ma anche per caratteristiche socio-ambientali, fa parte di quei territori che, seguendo la tipizzazione recentemente offerta da Mauro Varotto, è stata definita "montagna di mezzo". Un territorio paradigmatico delle aree interne italiane, in cui il forte spopolamento si associa ad una notevole carenza di servizi, ma in cui non mancano elementi di innovazione e dinamicità che contribuiscono a rendere Balme un paese turisticamente attrattivo (Del Bricco 2021). In questo contesto la Distilleria Quaglia ha saputo cogliere alcune delle opportunità inespresse di questo territorio periferico e marginale, valorizzandole attraverso la creazione di una filiera corta che dal campo e dal bosco conduce alla distilleria. Come ha efficacemente illustrato Varotto è oggigiorno necessario ed indispensabile un passaggio dal prodotto di montagna al prodotto della montagna, passando cioè da una generica geolocalizzazione aziendale a una più precisa definizione e indicazione relativamente a "provenienza di materie prime, tecniche di produzione, relazioni con ambiente, società e territorio» (Varotto 2020: 111). Non solamente si utilizzano materie prime del territorio per la produzione di liquori ma, attraverso la riorganizzazione della filiera, si ha una riattivazione di comunità che contribuisce ad attuare anche un complesso ed originale processo di patrimonializzazione e di valorizzazione delle risorse ambientali, che (ri)entrano così nel patrimonio gastronomico locale ${ }^{5}$.

21 Le erbe e i frutti coltivati nelle Valli di Lanzo contribuiscono non solo all'aromatizzazione del vermouth, ma danno anche vita, a partire da alcuni anni, a una specifica serie di prodotti d'eccellenza della Distilleria Quaglia, quali il Genepy di Balme, il Liquore alla Genziana e il Liquore al Timo Serpillo a cui si affianca il Genepy Piemonte IG, quest'ultimo contraddistinto dalla menzione di Indicazione Geografica (Mazzarino, Soster 2014; Vullo 2018: 171-172) e prodotto a partire da piante della specie Artemisia coltivate non solamente nell'areale delle Valli di Lanzo, ma nei territori alpini e appenninici dell'intero Piemonte. Si tratta di una serie di liquori che - riportando espressamente il nome di Balme e delle Valli di Lanzo in etichetta - concorrono alla definizione più complessiva dell'identità gastronomica contemporanea di questo territorio montano (Castagneri 2016). La produzione della distilleria Quaglia, a partire dalle parti aromatiche raccolte e coltivate nelle Valli di Lanzo, è solidamente inserita in una rete di rapporti sinergici e collaborativi con altre distillerie e altre realtà dell'eccellenza artigiana piemontese: è il caso ad esempio dell'Associazione per la tutela e la valorizzazione del Genepì (2002), dell'Istituto del Vermouth di Torino (2017) e del Consorzio del Vermouth di Torino (2019).

4. Nell'avviarsi alle conclusioni, è possibile affermare come il caso dei liquori alle erbe alpine prodotti dalla Distilleria Quaglia siano espressione di un più vasto fenomeno trasformativo - caratterizzato da «sostenibilità e saper fare nelle Alpi» (Bonato 2017) di cui è momento ineludibile il passaggio dal prodotto di montagna al prodotto della montagna cui già si è fatto riferimento, in cui «le montagne di mezzo possono ispirare il soddisfacimento di bisogni plurimi all'interno di un unico atto alimentare: il nutrimento, l'identità territoriale, la biodiversità agro-ecologica, la polifunzionalità di una pratica agricola capace di generare reddito e soddisfazione esistenziale, favorendo l'integrazione con altre attività economiche e piccole produzioni diversificate» (Varotto 2020: 119). Nel caso-studio preso in esame è proprio il lavoro attento fatto sulla filiera produttiva, rispettoso della sostenibilità ambientale e sociale nonché 
l'integrazione con l'economia del territorio che garantisce ulteriormente la "tipicità" del prodotto della montagna ${ }^{6}$.

È anche e soprattutto dal moltiplicarsi di esperienze come questa che si può invertire l'abbandono e tornare a «riabitare l'Italia» (De Rossi 2020; Cersosimo, Donzelli 2020). Con la creazione di microfiliere per la produzione di erbe officinali e di frutti destinati alla liquoristica nelle Valli di Lanzo trovano concreta applicazione e riscontro le considerazioni che circa vent'anni or sono Enrico Camanni faceva nel libro La nuova vita delle Alpi, laddove affermava che il riscatto dell'agricoltura di montagna fosse «ipotizzabile solo nei termini di una elevata qualità del prodotto e di una collocazione diretta sul mercato locale attraverso un circuito virtuoso con il mercato turistico: agriturismi, coltivazioni biologiche, marchi tipici, prodotti estremamente differenziati e assolutamente caratterizzati in base alla zona e addirittura all'azienda di provenienza. Non c'è alternativa. La montagna è costretta a seguire questa direzione» (Camanni 2002: 116).

24 La coltivazione delle erbe alpine a Balme può dunque essere considerata come un esempio emblematico e confermare come l'ipotesi auspicata da Camanni nel 2002 sia poi stata la strada, difficile ma vincente, verso un effettivo sviluppo sostenibile dei territori montani più fragili e periferici. Si tratta di territori che non hanno pressoché più nessun tipo di economia, dove il recupero di queste piccole produzioni e filiere diventa l'occasione non solamente per creare piccole ma redditizie attività economiche, ma anche per fare rete e creare comunità. La creatività di coltivare, nel profondo della provincia piemontese, erbe alpine laddove prima si faceva solo pascolo - è forse anche interpretabile come l'esito, imprevisto e imprevedibile, dello spopolamento dei decenni precedenti, del «vuoto» lasciato dalla generazione di chi ha abbandonato la montagna negli anni del miracolo economico della Nazione. Le storie dei ritornanti e dei neomontanari di Balme, la piccola storia delle erbe alpine e dei liquori della tradizione, «dimostrano che riempiendo i "vuoti" della montagna si può vivere bene, sicuramente meglio che in molte periferie urbane» (Dematteis 2020a: 167; Piacentini 2018: 15). È stato autorevolmente evidenziato come l'innovazione sociale e la creatività culturale abbiano necessità di trovare uno spazio entro cui esprimersi ${ }^{7}$ : «i soggetti non avrebbero modo di esprimere la creatività, se lacerazioni abbastanza consistenti non determinassero uno spazio disponibile» (Remotti 2011: 292). Questa ipotesi «sembra trovare conferma nelle Alpi italiane, dove si incontrano casi in cui il forte spopolamento ha consentito ai nuovi abitanti di approfittare del vuoto provocato da anni di emigrazione per avviare attività imprenditoriali in campo economico e anche culturale o comunque per inserirsi lavorativamente in modo innovativo in ambiti professionali non più presidiati dagli autoctoni» (Membretti, Ravazzoli 2020: 347). Se le cose stanno così, ecco allora che la visione suggerita dal poeta Franco Arminio - «l'Italia interna può diventare il laboratorio di un nuovo umanesimo, l'umanesimo delle montagne» (Arminio 2013: 22) - non è più solo un auspicio, ma una via concreta da perseguire per lo sviluppo delle aree montane italiane (Dematteis 2020b: 289-290). 


\section{BIBLIOGRAFIA}

Alberini M., 1967 Piemontesi a tavola. Itinerario gastronomico da Novara alle Alpi, Longanesi, Milano.

Arminio F., 2013 Geografia commossa dell'Italia interna, Bruno Mondadori, Milano.

Arpino G., Antonetto R. (a cura di), 1990 Torino altrui. Notazioni, giudizi, ricordi di forestieri su Torino, Famija Turinèisa, Torino.

Barbera F., Di Monaco R., Pilutti S., Sinibaldi E., 2019 Dall'alto in basso. Imprenditorialità diffusa nelle terre alte piemontesi, Rosenberg \& Sellier, Torino.

Bérard L., Marchenay P., 2004 Les produits de terroir. Entre cultures et règlements, Cnrs, Paris.

Berta P., Mainardi G., 1997 Piemonte, Unione italiana vini, Milano («Storia regionale della vite e del vino in Italia», 2).

Bertolino M.A., 2017 «Degli usi e delle virtù della lavanda. Agricoltura r-esistente e terzo paesaggio in aree marginali alpine: il caso della Valle Susa», in L. Bonato (a cura di), Aree marginali. Sostenibilità e saper fare nelle Alpi, Franco Angeli, Milano: 130-143.

Biasiol R., 1974 Piemonte in bottiglia ovvero come si beve in Piemonte e Valle d'Aosta, Eda, Torino. Bocchino A., 1988 Tante belle cose, Daumerie, Montiglio.

Bonato L. (a cura di), 2017 Aree marginali. Sostenibilità e saper fare nelle Alpi, Franco Angeli, Milano. Caffarena F., 2016 Scritture non comuni. Una fonte per la storia contemporanea, Unicopli, Milano.

Caire F., 1978 Cucina monferrina. Ricette tradizionali in uso nel casalese, Il portico, Casale Monferrato. Camanni E., 2002 La nuova vita delle Alpi, Bollati Boringhieri, Torino.

Camporesi P., 1998 Il brodo indiano. Edonismo ed esotismo nel Settecento, Garzanti, Milano.

Capatti A., Montanari M., 1999 La cucina italiana. Storia di una cultura, Laterza, Roma-Bari.

Carpignano N., 2017 Galuparie. Tradizioni culinarie del Piemonte dall'acciuga al verde allo zest di Carignano. Inventario sistematico dei prodotti tipici, ricette e curiosità del panorama enogastronomico regionale in oltre 1700 voci e 500 ricette, Daniela Piazza, Torino.

Castagneri G., 2016 Le radici del sapore. L'identità alimentare delle valli di Lanzo, Il Risveglio, Ciriè.

Castagneri G., Sguayzer M., 2010 Balme. Storia sociale di una comunità alpina (1308-2000), Uja, Lanzo Torinese.

Cavallero E., 2005 I malnutrì. Storia del cibo e della povertà in Piemonte attraverso 180 ricette dimenticate della cucina popolare, Daniela Piazza Editore, Torino.

Cavazza S., Scarpellini E. (a cura di), 2018 Storia d'Italia. Annali, 27, I consumi, Einaudi, Torino.

Cersosimo D., Donzelli C. (a cura di), 2020 Manifesto per riabitare l'Italia, con un dizionario di parole chiave, Donzelli, Roma.

Cipolla C., Damonti M. (a cura di), 2019 La grappa: il "grande" distillato italiano, Franco Angeli, Milano.

Cirese A.M., 1973 Cultura egemonica e culture subalterne. Rassegna degli studi sul mondo popolare tradizionale, $2^{\text {a }}$ ed., Palumbo, Palermo. 
Clavarino L., 1867 Saggio di corografia statistica e storica delle Valli di Lanzo, Stamperia della Gazzetta del popolo, Torino.

Club Amici Valchiusella (a cura di), 2010 El sabat d'le erbe: alla riscoperta delle erbe spontanee della Valchiusella, Club Amici Valchiusella, Traversella.

Club Amici Valchiusella (a cura di), 2011 El sabat d'le erbe: alla riscoperta delle erbe spontanee della Valchiusella sui sentieri della tradizione orientati al futuro, Club Amici Valchiusella, Traversella.

Corti M., 2011 I ribelli del bitto. Quando una tradizione casearia diventa eversiva, Slow Food Editore, Bra.

Dematteis G., 2020a «Montagna», in D. Cersosimo, C. Donzelli (a cura di), Manifesto per riabitare l'Italia, con un dizionario di parole chiave, Donzelli, Roma: 165-169.

Dematteis G., 2020b «Montagna e città: verso nuovi equilibri?», in A. De Rossi (a cura di), Riabitare l'Italia. Le aree interne tra abbandoni e riconquiste, nuova ed., Donzelli, Roma: 285-295.

De Rossi A. (a cura di), 2020 Riabitare l'Italia. Le aree interne tra abbandoni e riconquiste, Donzelli, Roma.

Del Bricco G., 2021 «Balme. Il decollo riuscito di un paese "Bombo"», in Passaggi e Sconfini, 4, n. 2: 19-23.

Dickie J., 2007 Con gusto. Storia degli italiani a tavola, Laterza, Roma-Bari.

Doglio S., 1995 Il dizionario di gastronomia del Piemonte, $2^{\text {a }}$ ed., Daumerie, Montiglio.

Fassino G., 2020 «Il caffè espresso: un tratto costitutivo della vita degli italiani», in Palaver, n.s., a. 9, n. 2: 167-188.

Fassino G., 2021 «I Granai della memoria: dal progetto al laboratorio», in D. Brunetti, D. Robotti, E. Salvalaggio (a cura di), Documenti sonori. Voce, suono, musica in archivi e raccolte, Regione Piemonte-Centro studi piemontesi, Torino: 403- 410.

Fassino G., Ghiardo L., 2016 Distilleria Quaglia. Sperimentare attraverso la tradizione: il vermouth, Università degli Studi di Scienze Gastronomiche - Laboratorio "Granai della memoria", PollenzoBra, video digitale, durata 8' 34" (disponibile online a questo link: www.ricerchedea.it/ videodea/).

Galiffa F., 2013 «Sui vini cotti dell'Abruzzo Teramano», in C. Cipolla, G. Di Francesco (a cura di), La ragion gastronomica, Franco Angeli, Milan: 87-116.

Gattullo M. (a cura di), 2002 Le confetture di Sua Maestà. Ricettario piemontese del XVIII secolo, Società per gli studi storici archeologici e artistici della provincia di Cuneo-Associazione Culturale Amici di Bene, Cuneo-Bene Vagienna.

Glielmi M.A., 2017 «Vivere la tradizione o tradurre l'arte di vivere? La raccolta della lavanda in Valle Gesso", in L. Bonato (a cura di), Aree marginali. Sostenibilità e saper fare nelle Alpi, Franco Angeli, Milano: 158-169.

Goria G., 1978 «Prefazione» a F. Caire, Cucina monferrina. Ricette tradizionali in uso nel casalese, Il portico, Casale Monferrato: 5-7.

Gozzano G., 1911 I colloqui. Liriche, Treves, Milano.

Grasseni C., 2007 La reinvenzione del cibo. Culture del gusto fra tradizione e globalizzazione ai piedi delle Alpi, QuiEdit, Verona.

Grasseni C., 2017 The heritage arena. Reinventing cheese in the Italian alps, Berghahn, New York. 
Grimaldi P., Fassino G., 2017 «Immaginari gastronomici / Gastronomic imagination», in A. Audisio (a cura di), Menu delle montagne, Priuli \& Verlucca, Scarmagno-Ivrea: 53-58.

Grimaldi P., Fassino G., Porporato D., 2019 Culture, Heritage, Identity and Food. A Methodological Approach, Franco Angeli, Milano.

Leitch A.P., 2003 «Ricordi del lardo / Memories of pork fat», in C. Barberis et al., Il Lardo di Colonnata. La via bianca del gusto tra i marmi di Carrara, Federico Motta, Milano: 110-143.

Mainardi G., Berta P. (a cura di), 2018 Il grande libro del vermouth di Torino. Storia e attualità di un classico prodotto piemontese, Oicce, Canelli.

Mazzarino S., Soster M., 2014 Il genepì: valutazioni economiche e organizzazione della microfiliera in Piemonte. Teoria e casi, Celid, Torino.

Membretti A., Ravazzoli E., 2020 «Immigrazione straniera e neo-popolamento nelle terre alte», in A. De Rossi (a cura di), Riabitare l'Italia. Le aree interne tra abbandoni e riconquiste, nuova ed., Donzelli, Roma: 333-349.

Montanari M., 2001 «Cucina povera, cucina ricca», in Quaderni medievali, LII: 95-105.

Montanari M., 2004 Il cibo come cultura, Laterza, Roma-Bari.

Odello L. et al., 2013 Atlante dei prodotti tipici. Grappe, acquaviti, liquori, Istituto Nazionale di Sociologia Rurale-Agra, Roma.

Perera O., 2001 Volti del gusto. Prodotti e produttori dell'artigianato alimentare, Musumeci, Quart.

Piacentini P., 2018 Appennino atto d'amore. La montagna a cui tutti apparteniamo, Terre di mezzo, Milano.

Piccinino F. (a cura di), 2016 Teodoro Negro e il suo Toccasana. Le Langhe tra storia e leggenda, L’Artistica, Savigliano.

Remotti F., 2011 Cultura. Dalla complessità all'impoverimento, Laterza, Roma-Bari.

Rosso C., 2009 «La cucina piemontese nel canone nazionale (secoli XVI-XX)», in G. Tesio (a cura di), Di cotte e di crude: cibo, culture, comunità. Atti del Convegno (Vercelli, 15-16 marzo 2007Pollenzo, 17 marzo 2007), Centro studi piemontesi, Torino: 53-71.

Ruggiero M., 2007 Piemonte. La storia a tavola. Le vicende dell'alimentazione, La bela Gigogin, Torino.

Scarpellini E., 2006 «L'utopia del consumo totale. L'evoluzione dei luoghi di consumo», in S.

Cavazza, E. Scarpellini (a cura di), Il secolo dei consumi. Dinamiche sociali nell'Europa del Novecento, Carocci, Roma: 21-50.

Scarpellini E., 2012 A tavola! Gli italiani in 7 pranzi, Laterza, Roma-Bari.

Serventi S. (a cura di), 1995 Il cuoco piemontese perfezionato a Parigi (Torino 1766), Slow Food Editore, Bra.

Varotto M., 2020 Montagne di mezzo. Una nuova geografia, Einaudi, Torino.

Vaudagnotto Bianco M.T., 2010 La cucina del risparmio, Priuli \& Verlucca, Scarmagno.

Venè G. F., 1990 Vola colomba. Vita quotidiana degli italiani negli anni del dopoguerra: 1945-1960, Mondadori, Milano.

Veronelli L. 1968 Piemonte, Valle d'Aosta, Garzanti, Milano.

Vialardi Giovanni, 1854 Trattato di cucina, pasticceria moderna, credenza e relativa confettureria, Favale, Torino. 
Viriglio A., 1898 Torino e i torinesi. Minuzie e memorie, Lattes, Torino.

Vullo S., 2018 Il Piemonte agricolo tra memoria e futuro, Nerosubianco, Cuneo.

Woolf S., Viazzo P.P. (a cura di), 2002 Formaggi e mercati. Economie d'alpeggio in Valle d'Aosta e HauteSavoie, Le Chateau, Aosta.

Zola L., 2013 «Dalle erbe spontanee al consorzio Erba Böna: saper e saper fare nelle valli Formazza e Antigorio», in L. Bonato, P.P. Viazzo (a cura di), Culture di confine. Ritualità, saperi e saper fare in Val D'Ossola e Valsesia, L’Artistica, Savigliano: 109-122.

\section{NOTE}

1. Attivato nell'ambito del corso di "Antropologia e memoria" (prof. Piercarlo Grimaldi e prof. Davide Porporato, aa.aa. 2015-16 e 2016-17). Primo esito di questa fase di lavoro è stato il videodocumentario Distilleria Quaglia. Sperimentare attraverso la tradizione: il vermouth (Fassino, Ghiardo 2016).

2. In particolare attraverso il portale web dei "Granai della memoria" (www.granaidellamemoria.it) sono disponibili le testimonianze di Carlo Quaglia, Federico Ricatto e Luca Fasano; sul progetto "Granai della memoria" cfr. Fassino 2021.

3. Questa citazione, così come le seguenti, è tratta dall'intervista a Carlo Quaglia realizzata nel 2017 e conservata nei "Granai della memoria", www.granaidellamemoria.it/index.php/it/ archivi/saperi-del-cibo/carlo-quaglia-2.

4. www.granaidellamemoria.it/index.php/it/archivi/saperi-del-cibo/federico-ricatto

5. Esistono nel territorio piemontese altre realtà in cui la filiera delle erbe spontanee ed officinali ha contribuito a rivitalizzare le reti comunitarie: è il caso ad esempio del consorzio Erba Böna nelle valli Antigorio e Formazza (Zola 2013), della coltivazione di lavanda in Valle di Susa e in Valle Gesso (Bertolino 2017; Glielmi 2017), della raccolta di erbe spontanee in Valchiusella (Club Amici Valchiusella 2010, 2011).

6. Per un confronto con altri casi-studio di produzioni alimentari di/della montagna italiana e francese cfr. Woolf, Viazzo 2002; Leitch 2003; Bérard, Marchenay 2004; Grasseni 2007, 2017; Corti 2011; Varotto 2020: 105-113.

7. Che le cose stiano così trova conferma del resto in numerose e diversificate testimonianze: l'innovazione creativa è infatti, più o meno consapevolmente, un tratto ricorrente nelle narrazioni di molti dei testimoni intervistati nel corso della ricerca Italian Mountain Lab - Ricerca e Innovazione per l'ambiente (se ne veda una selezione di videointerviste al sito del progetto https:// tourism.uniupo.it/progetti/italian-mountain-lab).

\section{RIASSUNTI}

L'articolo affronta il recente fenomeno del recupero dei liquori tradizionali, a partire dalla coltivazione di erbe officinali nelle Alpi piemontesi, attraverso l'analisi etnografica di una realtà imprenditoriale che negli ultimi anni ha contribuito in maniera significativa allo sviluppo di questa filiera. La ricerca sul campo ha indagato un'azienda che produce liquori e altri distillati a base di erbe di montagna coltivate nelle Valli di Lanzo (Torino). Un significativo esempio di come 
il recupero della coltivazione delle erbe officinali non solo mantenga viva una tradizione produttiva locale, ma contribuisca anche al più vasto fenomeno del riabitare le Alpi.

The article discusses the recent revival of traditional liqueurs, from the cultivation of medicinal herbs in the Piedmont Alps through the ethnographic analysis of the entrepreneurial activity that has contributed significantly to the growth of this market segment in recent years. Field research surveyed a company that produces liqueurs and other alpine herb-based spirits in the Valli di Lanzo (Torino). The company is a significant example of how the revival of cultivating medicinal herbs not only sustains a local productive tradition but also contributes to the greater trend of repopulating the Alps.

INDICE

Keywords : officinal plants, liqueurs, mountains, Graian Alps, gastronomic heritage

Parole chiave : erbe officinali, liquori, montagna, Alpi Graie, patrimonio gastronomico

\author{
AUTORE \\ GIANPAOLO FASSINO \\ Dipartimento di Studi Umanistici, Università degli Studi del Piemonte Orientale \\ gianpaolo.fassino@uniupo.it
}

